\title{
Implementation of Gaussian Process Regression in Estimating Reserve for Motor Vehicle Insurance Claims
}

\author{
Ria Novita Suwandani ${ }^{1}$, Yogo Purwono ${ }^{1}$ \\ Corresponding Email: rianovitasuwandani@gmail.com \\ ${ }^{1}$ Master of Management Program, Faculty of Economics and Business, University of \\ Indonesia
}

Received: January 6, 2021

Revised: January 27, 2021

Accepted: February 9, 2021

\begin{abstract}
This study aims to calculate the allowance for losses by applying Gaussian Process regression to estimate future claims. Modeling is done on motor vehicle insurance data. The data used in this study are historical data on the motor vehicle insurance business line of PT XYZ from January 2017 to December 2019. In estimating a period using the Gaussian Process method. The estimation results show that the Gaussian Process Regression method is very flexible and can be applied without much adjustment. These results were also compared with the Chain Ladder method.
\end{abstract}

Keywords: Claims Reserves, Gaussian Process Regression, Motor Vehicle Insurance

\section{Introduction}

The insurance industry is one of the sectors in the non-bank financial industry that plays an important role in supporting economic growth in Indonesia. In the general insurance industry, property insurance and motor vehicle insurance are the lines of business that provide the largest contribution to premium income. Based on data processed from the Insurance Statistics issued by financial fervices authority (OJK), the net premium for motor vehicle insurance compared to the total net premium from all general insurance business lines in the period 2015 - 2019 is more than 40\% (forty percent). Growth. This growth is expected to continue to increase because it is supported by growth in motor vehicle sales, where the average growth of motorized vehicles over the past five years has been 6.4\% (BPS, 2019).

The motor vehicle and property insurance business line is a relatively competitive business line for general insurance companies. General insurance companies in running their business, they have a tendency to try to offer premium rates that are relatively lower than other general insurance companies in the insurance industry (Brown \& Goolsbee, 2002). These lower rates are intended to attract prospective insured. This condition then led to a tariff war in the general insurance industry which then triggered unfair competition.

Many insurance companies put aside the principles of underwriting properly and prudently. This is not in accordance with the principles of a healthy insurance business where the level of premiums or contributions is sufficient, not excessive, and non-discriminatory. Therefore, to support the healthy growth of the insurance industry and prevent tariff wars between insurance companies, the Capital Market and Financial Institution Supervisory Agency (Bapepam-LK) as the regulator in the insurance sector considers it necessary to establish regulations regarding premium rate references that can be used as a reference. By industry in determining the amount of premium. 
Uncertainty in the timing of claims and losses that must be borne by an insurance company is closely related to the determination or prediction of a claim reserve. Claim reserve is very necessary for an insurance company to be able to run its insurance business properly. Insurance companies require a claim reservation to be able to make payments when a claim by the insured party or policy holder occurs. The claim reserve has a big influence on the health condition of the insurance company. The accuracy of the insurance company in calculating the estimated claim reserves can provide a positive value to the company's business, including in the determination of the appropriate product premium figure. An insurance company actuary is required to perform calculations in predicting the company's claim reserves. In general, in calculating claims reserves, the method used is derived from the run-off triangle data scheme. The method based on the run-off triangle scheme estimates future claims projections by compiling historical data into a triangle and then analyzing it.

According to Antonio et al (2006), the run-off triangle data is a description of aggregate claims and is a summary of individual claims data. The most basic calculation in determining claims reserves is deterministic calculation, one of which is by using the Chain-Ladder method. The Chain-Ladder method calculates the claim reserves based on the run-off triangle scheme on the cumulative data. According to Hürlimann (2009), Gigante et al (2013), Wüthrich \& Merz (2008), using the Chain-Ladder method will make it easier and simpler to estimate claims reserves.

Seeing the importance of calculating claims reserves for insurance companies, research is conducted to calculate the prediction of claims reserves. Such as research conducted by Taylor (2012) and Wüthrich \& Merz (2008) which examines the prediction of claims reserves and associated variability. Another study concerning the Bayesian Model researched by De Alba \& Nieto-Barajas (2008). Furthermore, Shi \& Hartman (2016) explain the same correlation using the Bayesian hierarchical model.

To complement the existing methods for estimating allowance for losses, I researched the Gaussian Process method. The Gaussian Process method was chosen because the Gaussian Process method is a flexible, nonparametric statistical method that provides a good fit for various types of data, structures, and distributions and produces better predictions than the Chain Ladder method.

In addition, according to Lally \& Hartmman (2018) the Gaussian Process method is a nonparametric method, so that the relationship between the year of occurrence, development delay, and losses is studied from existing data. And there is no need for post-hoc adjustments, namely looking for which variables have a significant difference. Post-hoc adjustments are usually used in deterministic methods. Through its covariance function, Gaussian Process regression naturally models the dependency structure between losses in both the accident period dimensions and development delays. Gaussian Process Regression only requires the estimation of a few hyperparameters to study the complex relationships that exist in the data.

However, the Gaussian Process method is not a stand-alone method, it only adjusts the residuals in the Chain Ladder calculation to get a more accurate prediction of IBNR claim reserves.

Thus, this study aims to apply the Bayesian Gaussian Process Regression model in calculating the estimated IBNR claim reserves in the context of motor vehicle insurance in Indonesia. Therefore, this study aims to apply the Gaussian Process Regression method in estimating the IBNR claim reserves in the motor insurance business line of PT. Asuransi $\mathrm{XYZ}$ and compares the estimation results of IBNR claims reserves in the motor vehicle 
insurance business line of PT Asuransi XYZ between the Gaussian Process Regression method and the classic Chain-Ladder method.

\section{Reserve Claims}

The Financial Services Authority (OJK, 2018) explained about the financial health of insurance companies and reinsurance companies that the financial health of insurance companies is determined by several factors, one of which is technical reserves. Technical reserves are calculated as company liabilities and consist of several parts, one of which is reserves for claims. To calculate the amount of the prepared claim reserves, at least it is calculated equal to the sum of the estimated value of claims that are in the process of settlement (RBNS / Reported But Not Settled) and the estimated value of claims that have occurred but have not yet been reported (IBNR / Incurred But Not Reported).

Explanation of the RBNS (Reported but Not Settled) claim referring to Wüthrich \& Merz (2008) is the claim value that has been entered into the record or has been reported but has not been paid during the reporting period of the accounting record. Wüthrich \& Merz (2008) provide another definition for the RBNS claim as IBNeR (Incurred but Not enough Reported).

The regulation regarding claims reserves issued by the chairman of the Capital Market and Financial Institution Supervisory Agency in 2012 (OJK, 2012) issued regulations regarding technical reserves for claims reserves as follows: (1) Technical reserves in the form of claims reserves are calculated at least equal to the sum of the estimated claim values still in the process of being completed; estimated claims incurred but not reported.

The value of claims for insurance products and or reinsurance products that are still in the stage of completion is at least calculated based on a central estimate or best estimate for claims that have occurred and have been reported but are still in the process of settlement, together with the calculation of costs for insurance loss adjudication services, costs. Legal settlement and other costs associated with settling claims processing.

The value of claims that have occurred but have not been reported (Incurred but Not Reported) is calculated based on the central estimate or best estimate for claims that have occurred but have not been reported using the claim ratio method or one of the triangle methods, along with cost of insurance loss appraisal services.

Regarding claims reserves in the process as referred to in point (1) letter a cannot / can be estimated, the amount to be reserved is the average percentage of claims paid against the sum insured for the same line of business in the last financial year period multiplied by the sum insured from these claims.

The discussion regarding claim reserves is also related to the process of how these claims occur. Wüthrich \& Merz (2008) describes the claim process in general as follows:

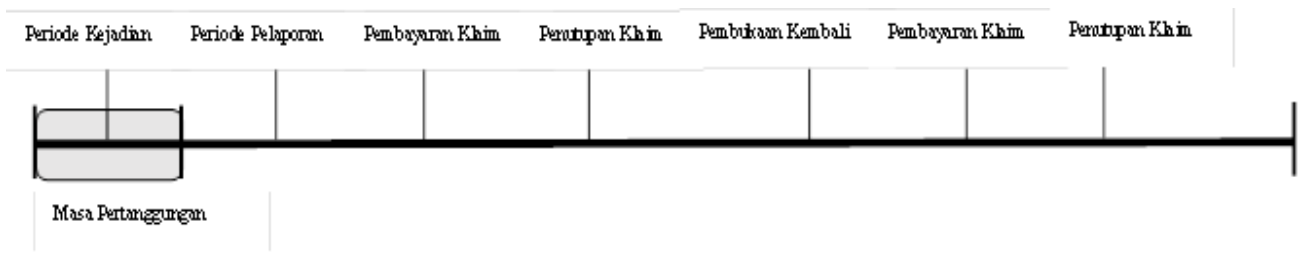

Figure 1. Schematic of General Claims Process

Source: Wüthrich \& Merz (2008)

Copyright ( 9 2021, Journal of Asian Multicultural Research for Economy and Management Study,

Under the license CC BY-SA 4.0 
Explanation of the claim process is based on Figure 1. The first step in a claim is an accident (during the insurance contract period). When this first stage occurs, the insurer does not know or receive a report from the insured regarding the accident. Furthermore, the second stage is that the accident is reported to the insurer (insurance company) and at this stage the insurance company records the incident but has not made any payment or compensation to the insured party. The third stage is the payment of claims by the insurer. It is at this stage that the insurance company makes a payment or replaces an amount with the value specified in the insurance contract with the insured party. The amount of the amount of the payment or compensation value is adjusted to the value of the object insured by the insured party. The last stage is closing the claim. Determination of this claim is addressed to the claim process that has been paid to the insured party. The next stage is the re-opening of the claim, this stage will only be carried out if there is a request or obligation from the insured party or the insurer that has not been resolved. It should be noted, at the reporting and payment stages of claims there is a gap between reporting and payment, this is known as the lag period for claim payments.

\section{Claim Reserves Analysis Method}

In analyzing the reserves of claims, various methods of estimating reserves can be done. The method of analyzing claims reserves is generally divided into two broad lines, namely the deterministic and stochastic methods. The deterministic method that is often used by practitioners in analyzing claims reserves is the Chain-Ladder method developed by Mack \& Nielsen (1993). The Chain-Ladder method certainly has shortcomings which will be corrected by several alternative methods, namely the Generelized Estimating Equations method by Hudecová \& Pešta (2013) which is used to estimate claims reserves where claims at different origins and development periods do not have to be independent, The Double Chain-Ladder Method Martínez-Miranda et al (2012) is used because it can separate the value of the RBNS and IBNR claims reserves, the Munich Chain-Ladder method by Jedlicka (2007) which uses the residual correlation between paid claims and reported claims by calculating parallel data in the past to project into the future.

\section{Chain-Ladder Method}

The Chain-Ladder method is widely recognized as an actuarial method in calculating estimated claims reserves because it is an easy method to apply. The calculation of future claims using the Chain-Ladder method is based on the assumption that claims that will occur in the future are carried out in the same way as claims that have occurred.

Suppose that $S_{i j}$ is the cumulative claim value in the event year $i$, for $i=1,2,3, \ldots, n$ paid up to the development period $j$ for $j=1,2,3, \ldots n$. $S_{i j}$ considered as an observable random variable (run-off triangles), if $i=1,2,3, \ldots, n$ and $j=1,2,3, \ldots, n-1+1$. The goal to be achieved is to estimate the total (ultimate) claim $S_{i j}$, and the unpaid (payable) claims reserve:

$$
R_{i}=S_{1, n}-S_{i, n+1-1}, \text { for the event period } i=2, \ldots, n
$$

According Mack \& Nielsen (1993) Basic assumptions of the method Chain-Ladder is there development factor:

$\lambda_{1}, \lambda_{2}, \ldots, \lambda_{n-1}>0$, with:

$$
\begin{gathered}
E\left(C_{i, j+1} \mid C_{i, 1}, C_{i, 2}, \ldots, C_{i, j}\right)=C_{i, j} \cdot \lambda_{j}, \text { for } i=1,2,3, \ldots, n \text { and } \\
j=1,2,3, \ldots, n-1
\end{gathered}
$$


with estimated factors $\lambda_{j}$, using a formula:

$$
\hat{\lambda}_{j}=\frac{\sum_{n=1}^{n-j} C_{i, j+1}}{\sum_{n=1}^{n-j} C_{i j}}
$$

For $j=1,2, \ldots, n-1$

For the total claim $S_{i, n}$ by formula:

$$
\hat{S}_{i, n}=C_{i, n+1-i} \cdot \hat{\lambda}_{n+1,-i} \cdots \cdot . \hat{\lambda}_{n-i}
$$

or backup $R_{i}$ by formula:

$$
\hat{R}_{i}=C_{i, n+1-i} \cdot\left(\hat{\lambda}_{n+1-i} \cdots \cdot \hat{\lambda}_{n-i}-1\right)
$$

Since the Chain-Ladder algorithm does not take into account the dependence between accident years, it is an additional assumption that the variable $S_{i j}$ for different accident years $\left\{S_{i, 1}, \ldots, S_{i, n}\right\},\left\{S_{i, 1}, \ldots, S_{i, n}\right\}, i \neq j$ is independent.

This can further be considered as the absolute assumption of the Chain-Ladder method. In practice, the irrelevance between accident periods can be distorted by the effects of certain periods, such as changes in the way claims are settled or in the method of backing up claims.

\section{Gaussian Process Regression Method}

According to Lally \& Hartman (2018), the Gaussian Process can be assumed to have a matrix $X \in \mathbb{R}^{n . p}$ and vectors $Y \in \mathbb{R}^{n}$, Gaussian Process regression can be applied to study unknown functions $f(x)$ (where $X \in \mathbb{R}^{p}$ is any row vector $X$ ) that models the target $y$.

The Gaussian process can be used in regression modeling. The purpose of regression modeling is to determine the relationship between several variables. Gaussian Process Regression aims to estimate the function that can relate a known input set to an output series.

\section{Stochastic Process}

Stochastic processes are mathematical objects, defined as families of random variables. Many stochastic processes can be represented by a time series. However, stochastic processes are basically continuous whereas time series is a set of observations indexed by integers. Stochastic processes may involve several related random variables.

\section{Gaussian Process}

The Gasussian process is a stochastic process of a finite set of random variables that has a multivariate normal distribution, where each linear combination of these variables has a normal distribution. According to Lally \& Hartman (2018) for all subsets of the point time distribution $Y_{t}=\left\{t_{1}, t_{2}, \ldots, t_{m}\right\}$ in $\Omega$, a process $\left\{Y_{t}\right\} t \in S$ is Gaussian if $\left\{Y_{t 1}, Y_{t 2}, \ldots, Y_{t m}\right\}$ is the multivariate Gaussian.

The Bayesian Gaussian process can be expressed by the following, $f \sim N_{n}(M(X), K(X, X))$ for individual input pairs $\mathrm{x}$ and $\mathrm{x}$ 'where $(x)=\mathbb{E}[f(x)]$ $k\left(x, x^{\prime}\right)=\mathbb{E}\left[(f(x)-m(x))\left(f\left(x^{\prime}\right)-m\left(x^{\prime}\right)\right)\right], \quad f(x) \sim \mathcal{G P}\left(m(x), k\left(x, x^{\prime}\right)(2.8)\right.$

For simplicity, the mean chosen for the Gaussian Process Regression is $M(X)=0$. Although the assumption of a mean-value function is somewhat unlikely to be the case, it can still be used. If the response variable is standardized, then each output from the process lies around 
the observed mean. The Gaussian process is by no means limited to having a mean of zero. Alternatively, one can select any function that satisfies the given mapping.

\section{Covariance Functions}

One of the important processes in the Gaussian Process regression is the selection of covariance functions or what is often called the kernel function. In Lally \& Hartman's (2018) study, 3 covariance functions were used, namely, the Matern covariance function $3 / 2$, the Matern covariance function $5 / 2$ and quadratic exponential covariance function. In this study, only one of the covariance functions is discussed, namely the quadratic exponential covariance function.

\section{Stationarity and Isotropic}

The covariance functions used are stationary and isotropic. Menutut Barber (2012) variables $x$ as input to a one-dimensional Gaussian process, $x_{p}$ and $x_{q}$ are two dots on $x$, covariance function of $\left(x_{p}, x_{q}\right)$ is stationarity if the covariance function consists of splitting $x_{p}-x_{q}$. So that $k\left(x_{p}, x_{q}\right)=k\left(x_{p}-x_{q}\right)$. And the covariance function is isotropic if it is a function of distance $d=\left|x_{p}-x_{q}\right|$. The covariant function is by construction rotationally invariant.

The Euclidean distance between 2 data points to be calculated and used as calculation input is $x_{1}$ (development lag) and $x_{2}$ (accident period). This distance will be used as input for the covariance function. Thus, the covariance function is a function of stationarity and isotropic covariance.

Long scale characteristics $(l)$ defined to apply to the covariance function. The length scale determines how far a point needs to move along a certain axis so that the function value is uncorrelated. Giraud et al (2016).

In Lally \& Hartman's (2018) research, parameters are introduced bandwith $\psi$, where $\psi=\frac{1}{2 l^{2}}$ to implement the length scale in the Gaussian process regression. A large value of 1 yields $\psi$ small ones, and vice versa. Bandwidth parameter is defined separately for each variable $x_{1}$ and $x_{2}$, is $\psi_{1}$ and $\psi_{2}$.

\section{Signal and Noise}

The covariance function used in this study is the normalized covariance function. Then, if the distance between $x_{p}$ and $x_{q}$ is 0 , so $k\left(x_{p}, x_{q}\right)=1$ for all functions. It assumes that the underlying process has a variant 1 . To relax this assumption, Lally \& Hartman (2018) introduced the parameter $\eta^{2}$ for all covariance functions and multiplied by the covariance function.

This calculation of the covariance function allows a lot of noise. Therefore, Lally \& Hartman (2018) introduced parameters $\delta_{p, q}$ to the covariance function. Where $\delta_{p, q}$ is delta Kronecker $\left(\delta_{p, q}=1\right.$ if $p=q$ and 0 if no). So that the quadratic covariance function that will be used in the study becomes:

$$
k\left(x_{p}, x_{q}\right)=\eta^{2} \exp \left(-d^{2}\right)+\sigma^{2} \delta_{p, q}
$$

\section{Input Warping}

Input Warping is a methodology that can be used to improve the results of non-stationary processes which are modeled with a stationary covariance function, as defined by Snoek et al (2014). 
Lally \& Hartman (2018) in their research have determined that the Gaussian process regression in calculating the allowance for losses requires Input Warping correction in the model. Input Warping, converts non-stationary processes to stationary by changing the covariance function of $k\left(x_{p}, x_{q}\right)$ become $k\left(w\left(x_{p}\right), w\left(x_{q}\right)\right)$. Function $w(x)$ is a warping function that modifies the input vector into a stationary vector (Snoek et al., 2014). In theory, many warping functions can be applied, but Snoek et al (2014) recommends using the Beta Commulative Distribution Function (CDF). Because the Beta function can take many forms, for example linear, exponential, logarithmic by varying two parameters $\alpha$ and $\beta$. Because the Beta distribution is only valuable $[0,1]$, vektor input dinormalisasi ke interval $[0,1]$. For the model used, wrapping is done on the input vector $x_{1}$ and $x_{2}$ with its own warping function. Therefore, the input data is redefined as follows:

$$
\begin{aligned}
& w_{1}(x)=\operatorname{BetaCDF}\left(x, \alpha_{1}, \beta_{1}\right) \\
& w_{2}(x)=\operatorname{BetaCDF}\left(x, \alpha_{2}, \beta_{2}\right)
\end{aligned}
$$

\section{Hyperparameters and Prior Distribution}

All hyperparameters that will be applied in the model have been discussed in the previous section. To make adequate conclusions from each hyperparameter, the prior distribution for each hyperparameter needs to be known where it is determined. In Lally \& Hartman's (2018) model, the prior distribution has been determined for each parameter and can be explained in the following table.

Table 1. Hyperparameters and Prior Distribution in Gaussian Process Regression

\begin{tabular}{|c|l|l|}
\hline Parameter & \multicolumn{1}{|c|}{ Description } & Distribusi Prior \\
\hline $\boldsymbol{\psi}_{\mathbf{1}}$ & Bandwith parameter DL-Axis & gamma $(4,4)$ \\
\hline $\boldsymbol{\psi}_{\mathbf{2}} \cdot$ & Bandwith parameter IY-Axis & gamma $(4,4)$ \\
\hline $\boldsymbol{\eta}^{\mathbf{2}}$ & Signal parameter & $T^{+}(4,0,1)$ \\
\hline $\boldsymbol{\sigma}^{\mathbf{2}}$ & Noise parameter & $T^{+}(4,0,1)$ \\
\hline $\boldsymbol{\alpha}_{\mathbf{1}}$ & Alpha parameter of the warping function DL-Ax & $\ln N(0,0.5)$ \\
\hline $\boldsymbol{\beta}_{\mathbf{1}}$ & $\begin{array}{l}\text { Beta parameters of the DL-Axis warping } \\
\text { function }\end{array}$ & $\ln N(0,0.5)$ \\
\hline $\boldsymbol{\alpha}_{\mathbf{2}}$ & $\begin{array}{l}\text { Alpha parameter of the IY-Axis warping } \\
\text { function }\end{array}$ & $\ln N(0,0.5)$ \\
\hline $\boldsymbol{\beta}_{\mathbf{2}}$ & $\begin{array}{l}\text { Beta parameter of the IY-Axis warping } \\
\text { function }\end{array}$ & $\ln N(0,0.5)$ \\
\hline
\end{tabular}

Source: Ruitenberg (2019)

\section{Methods}

The data used in this study are historical data on PT XYZ's motor vehicle insurance business line during 2017 and 2019 (January 2017 to December 2019). The use of 2017-2019 data is due to the availability of the latest data from the company. The data taken is the overall data in the motor vehicle insurance business line of PT XYZ, the data consists of the insurance policy, cause of loss, date of incident, date of coverage, date of claim payment, amount of claim submitted, amount of claim paid by PT XYZ. The data is then grouped into months, this is because the business lines in motor vehicle insurance have a short-tail business class,

Copyright (C) 2021, Journal of Asian Multicultural Research for Economy and Management Study, 
where the delay between the time a claim occurs and the time for claim settlement has a short period, usually less than one year ( Larhed \& Olofsson, 2006; Freidland, 2010).

Data analysis will be carried out on the 2017-2019 data to obtain estimated claims reserves for the following year, namely 2018 - 2020. In assessing the accuracy of the estimated claims reserves, data for 2017-2019 obtained from the company will be used as actual data for compared with the estimated value in the Gaussian Process method.

\section{Gaussian Process Method}

In estimating a period using the Gaussian Process method, the first step that must be taken is to prepare data on claims that have occurred in the previous period in the form of an incremental triangle run-off. From the calculation of the incremental run-off form, the claim data is obtained for each time period.

The Gaussian Process Method in this study which follows the process in Lally \& Hartman (2018) defines 2 input variables, namely:

$$
\begin{aligned}
& x_{1}=\text { time the claim is paid (development period) } \\
& x_{2}=\text { time of claim (period of incident) } \\
& Z=\text { the kalim value for the period }
\end{aligned}
$$

Both variables are calculated in months.

\section{Stages of the Calculation Process Gaussian Process Regression Method}

The steps taken in calculating the Root Mean Square Error of Prediction (RMSE) from the Gaussian Bayesian Process method are described as follows:

\section{Defines the input variable.}

The input variable is $x$ and $z$. Where $x_{1}$ is development lag dan $x_{2}$ is accident period. $Z$ is the number of claims.

Normalization of the input variables for the covariance function. As explained in point 1 , there are 2 input variables. With these two input parameters, the euclidean distance between these 2 distance points is used as a parameter of the covariance function. With the run off triangle data that has been obtained previously, the variables $x_{1}$ and $x_{2}$ can be normalized with the following formula:

$$
x^{\prime}=\frac{x-\min (x)}{\max (x)-\min (x)},
$$

\section{Standardization of Claim Value $(Z)$}

Once obtained $x_{1}^{\prime}$ and $x_{2}^{\prime}$, the next step that must be done is to standardize the claim value using the following formula:

$$
z_{i}^{\prime}=\frac{z_{i}-\bar{Z}}{s}, \text { for all } i \in\{1,2, \ldots, n\}
$$

Where $\bar{Z}=$ mean from $Z$ and $s$ is the standard deviation of $Z$. The following are the results of standardization $Z$ using a formula (3.12)

\section{Select the covariance function to perform data input}

According to Lally \& Hartman (2018) there are 3 types of covariance functions that can be used, namely the Matern 3/2 covariance function, the Matern 5/2 covariance function and the quadratic exponential covariance function with the following function formula 
Table 2. Covariance Functions Matern 3/2, Matern 5/2 and Exponential Quadratic

\begin{tabular}{ll}
\hline Covariance Function Name & Equation \\
\hline Matérn 3/2 & $\eta^{2}(1+\sqrt{3} d) \exp (-\sqrt{3} d)$ \\
Matérn 5/2 & $\eta^{2}\left(1+\sqrt{5} d+\frac{5}{3} d^{2}\right) \exp (-\sqrt{5} d)$ \\
Squared Exponential & $\eta^{2} \exp \left(-d^{2}\right)$ \\
\hline
\end{tabular}

In this paper we will only use the quadratic exponential covariance function, which is as follows:

$$
k\left(x, x^{\prime}\right)=\eta^{2} \exp \left(d^{2}\right)
$$

Where $d^{2}=\left(x-x^{\prime}\right)^{T} \psi\left(x-x^{\prime}\right)$ and $\psi=\operatorname{diag}\left(\psi_{1}, \psi_{2}\right)$

\section{Input Parameters}

Prior parameter used is $\alpha_{1}, \beta_{1}, \alpha_{2}, \beta_{2}$ which reflects the form of stationarity. While the hyperparameter used is log-normal, which is as follows:

$$
\begin{aligned}
& \alpha_{j} \sim \ln \mathcal{N}(0,0.5) \\
& \beta_{j} \sim \ln \mathcal{N}(0,0.5)
\end{aligned}
$$

untuk $j \in\{1,2\}$. Sedangkan hyperparameter untuk bandwith, noisy varian dan signal varian adalah

$$
\begin{gathered}
\sigma^{2} \sim \mathcal{T}^{+}(4,0,1) \\
\eta^{2} \sim \mathcal{T}^{+}(4,0,1) \\
\psi_{1} \sim \operatorname{gamma}(4,4) \\
\psi_{2} \sim \operatorname{gamma}(4,4)
\end{gathered}
$$

\section{Calculating Root Mean Square Error (RMSE)}

The estimated claim reserves obtained from the Chain Ladder method will be compared with the Gaussian regression process that occurred during 2017 - 2019. To get a good comparison result, a Root Mean Square Error (RMSE) test will be carried out. The following is the formulation of RMSE

$$
R M S E=\frac{\sum_{t=1}^{T}\left(\widehat{Z}_{t}-Z_{t}\right)^{2}}{T}
$$

\begin{tabular}{|c|c|c|c|c|c|c|}
\hline \multicolumn{7}{|c|}{ Estimated reserves for claims } \\
\hline \multirow{2}{*}{$\begin{array}{l}\text { the period of } \\
\text { the incident }\end{array}$} & \multicolumn{2}{|c|}{2017} & \multicolumn{2}{|c|}{2018} & & \\
\hline & $\mathrm{CL}$ & GP & CL & GP & CL & GP \\
\hline
\end{tabular}

Where $Z_{t}=$ estimated data and $T=$ Amount of estimated data

\section{Results and Discussion}

From the calculation of the estimated claim reserves using the Chain Ladder and Gaussian Process Regression method in the motor vehicle insurance business line of PT XYZ in 2017 2019, the following results are obtained:

Table 2. Estimation Result of Claim Reserves 


\begin{tabular}{|c|c|c|c|c|c|c|}
\hline 2 & $\begin{array}{c}639.811 . \\
984\end{array}$ & 644.290 .668 & 2662.319 .062 & 2.660 .955 .295 & 3.916 .821 & 1.561289 \\
\hline 3 & $\begin{array}{c}871428.6 \\
18\end{array}$ & 877.528 .619 & 2.470 .138 .836 & 2.487 .427 .429 .807 & 10.578 .578 & 10.652 .628 \\
\hline 4 & $\begin{array}{c}477.507 . \\
167\end{array}$ & 480.849 .717 & 3.046 .227 .788 & 3.067 .551 .382 & 11.0200 .080 & 11.097 .211 \\
\hline 5 & $\begin{array}{c}176.844 . \\
948\end{array}$ & 178.082 .862 & 1.394 .658996 & 1.404 .421 .609 & 18.788 .461 & 18.919 .981 \\
\hline 6 & $\begin{array}{c}105.116 \\
203\end{array}$ & 105.852 .016 & 1.650 .665 .396 & 1.662 .220 .054 & 54.822 .422 & 55.206 .179 \\
\hline 7 & $\begin{array}{c}343.360 \\
263\end{array}$ & 345.763 .785 & 1.315 .200 .978 & 1.324 .407 .385 & 65.439 .005 & 66.897 .078 \\
\hline 8 & $\begin{array}{c}576.385 \\
432\end{array}$ & 580.420 .130 & 1.060 .367 .730 & 1.067 .790 .305 & 111.972 .046 & 112.755 .850 \\
\hline 9 & $\begin{array}{c}487.615 \\
721\end{array}$ & 491.029 .031 & 727.258 .532 & 732.349 .341 & 146.936 .777 & 147.965 .334 \\
\hline 10 & $\begin{array}{c}603.875 \\
921\end{array}$ & 608.108 .053 & 732.864 .389 & 737.994 .439 & 257.702 .882 & 259.506 .802 \\
\hline 11 & $\begin{array}{c}2.405 .54 \\
8.745\end{array}$ & 2.422 .387 .586 & 973.119947 & 979.931 .787 & 484.482 .699 & 487.874 .007 \\
\hline 12 & $\begin{array}{c}2310.484 \\
.221\end{array}$ & 2.326 .657 .610 & 161.861 .951 & 162.813 .725 & 554.104 .748 & 557.983 .481 \\
\hline Total & $\begin{array}{c}8.997 .97 \\
9.222\end{array}$ & 9.060 .966 .077 & 16.194 .503 .605 & 16.307 .865 .130 & 1.719 .764 .519 & 1.729 .419 .920 \\
\hline
\end{tabular}

In estimating the claim reserves using the Chain Ladder, the results are Rp. 8,997,979,222 in 2017, Rp. 9,060,965,077 in 2018, Rp. 1,719,764,519 in 2019 and the calculation of estimated claim reserves using the Gaussian process method yielded Rp. 9,060,965,077 in 2017, Rp. $16,307,865,130$ in 2018, and Rp. $1,729,419,920$ in 2019. The backup results of these two methods will be compared with the Root Mean Squaree Error (RMSE). The results of the RMSE are as follows:

Table 3. RMSE Result

\begin{tabular}{|c|c|c|c|}
\hline & $\mathbf{2 0 1 9}$ & $\mathbf{2 0 1 8}$ & $\mathbf{2 0 1 7}$ \\
\hline CL & 710.220 & $5 . .619 .036$ & 1.350. \\
\hline Quadratic exponential & 22,327 & 5.213 .424 & 1.839 .219 \\
\hline
\end{tabular}

The results obtained from the RSME calculation show that the prediction of claim reserves for 2018 and 2019 is smaller and tends to be better.

\section{Conclusion}

Estimated claim reserves for PT XYZ's motor vehicle business line using the chain-ladder method, the company must provide funds for 2017 of IDR 8,997,979,222, in 2018 IDR. Rp. 16,194,503,605, in 2019 amounting to Rp. 1,719,764,520 for backup. Meanwhile, by using the Bayessian Gaussian Process method, the company must provide funds for 2017 of Rp. 9,060,965,077, in 2018 amounting to Rp. 16,307,865,130 and in 2019 Rp. 1,731,802,871 for backup.

\section{References}

Antonio, K., Beirlant, J., Hoedemakers, T \& Verlaak, R. (2006). Lognormal mixed models for reported claim reserve. North American Actuarial Journal 10(1),30-48.

Barber, D. (2012). Bayesian reasoning and machine learning. Cambridge University Press.

Copyright $@$ C 2021, Journal of Asian Multicultural Research for Economy and Management Study,

Under the license CC BY-SA 4.0 
BPS. (2019). Perkembangan Jumlah Kendaraan Bermotor Menurut Jenis, 1949-2018. https://www.bps.go.id/linkTableDinamis/view/id/1133

Brown, J. R., \& Goolsbee, A. (2002). Does the Internet make markets more competitive? Evidence from the life insurance industry. Journal of political economy, 110(3), 481-507.

de Alba, E., \& Nieto-Barajas, L. E. (2008). Claims reserving: a correlated Bayesian model. Insurance: Mathematics and Economics, 43(3), 368-376.

Friedland, F., \& Chasan, M. B. (2010). U.S. Patent Application No. 12/258,559.

Gigante, P., Picech, L \& Sigalotti, L. (2013). Prediction error for credible claims reserves: an h-likelihood approach. European Actuarial Journal, 3(2),453-470.

Giraud, W. J., Kingsbury, B. D., Rasmussen, M. H., \& Rodriguez, D. (2016). U.S. Patent No. 9,519,118. Washington, DC: U.S. Patent and Trademark Office.

Hudecová, Š., \& Pešta, M. (2013). Modeling dependencies in claims reserving with GEE. Insurance: Mathematics and Economics, 53(3), 786-794.

Hürlimann, W. (2009) Credible loss ratio claims reserves: The Benktander, Neuhaus and Mack methods revisited. Astin Bulletin, 39(01).

Jedlicka, P. (2007). Various extensions based on Munich Chain Ladder method. In ASTIN Colloquium, Orlando, Florida, http://www. actuaries. org/ASTIN/Colloquia/Orlando/Presentations/Jedlicka. pdf.

Lally, N., \& Hartman, B. (2018). Estimating loss reserves using hierarchical Bayesian Gaussian process regression with input warping. Insurance: Mathematics and Economics, 82, 124-140.

Larhed, M., \& Olofsson, K. (Eds.). (2006). Microwave methods in organic synthesis (Vol. 266). Springer.

Mack, R., \& Nielsen, J. (1993). Usability inspection methods: Report on a workshop held at CHI'92, Monterey, CA, May 3-4, 1992. ACM Sigchi Bulletin, 25(1), 28-33.

Martínez-Miranda, M. D., Nielsen, J. P., \& Verrall, R. J. (2012). Double chain ladder. Astin Bulletin, 42(1), 59-76.

Shi, P., \& Hartman, B. M. (2016). Credibility in loss reserving. North American Actuarial Journal, 20(2), 114-132.

Snoek, J., Swersky, K., Zemel, R., \& Adams, R. (2014, June). Input warping for bayesian optimization of non-stationary functions. In International Conference on Machine Learning (pp. 1674-1682). PMLR.

Taylor, G., McGuire, G., \& Greenfield, A. (2003). Loss reserving: past, present and future. University of Melbourne Centre of Actuarial Studies Research Paper, (109).

Wüthrich, M.V \& Merz, M. (2008). Stochastic Claims Reserving Methods in Insurance. In: Wiley Finance Series, John Wiley \& Sons. 